SELECCIONES MATEMÁTICAS
Universidad Nacional de Trujillo
ISSN: 2411-1783 (Online)
Vol. 06(01): $1-13(2019)$

\title{
Mathematical models for Zika with exposed variables and delay. Comparison and experimentation in Suriname and El Salvador.
}

\section{Modelos Matemáticos para Zika con Variables Expuesto y retardo. Comparación y Experimentación en Surinam y El Salvador.}

\author{
Erick Manuel Delgado Moya ${ }^{*}$ and Aymee Marrero Severo ${ }^{\dagger}$ \\ Received, Dec. 26, 2018 \\ Accepted, May. 11, 2019
}

DOI: http://dx.doi.org/10.17268/sel.mat.2019.01.01

\begin{abstract}
The Zika Virus (ZIKV) is a virus transmitted by Aedes aegypti mosquitoes (same as the one transmitting dengue and chikungunya fever). The main way of contagion by the ZIKV is caused by the bite of a mosquito that, after feeding from someone contaminated, can transport the virus throughout its life, transmitting the disease to a population that does not have the immunity. It can also be transmitted through a person's sexual relationship with ZIKV to their partners, even if the infected person does not have the symptoms of the disease. In this work, we present two mathematical models for the Zika epidemic by using (1) ordinary differential equations and, (2) ordinary differential equations with temporal delay (discrete), which is the time it takes mosquitoes to develop the virus. We make a comparison between the two modeling variants. Computational simulations are performed for Suriname and El Salvador, which are countries that are prone to develop the epidemic in an endemic manner.
\end{abstract}

Keywords. Ordinary differential equations, models, delay, transmission, ZIKV.

\section{Resumen}

El virus Zika (ZIKV) es un virus transmitido por los mosquitos Aedes aegypti (igual que el que transmite el dengue y la fiebre chikungunya). La principal forma de contagio por el ZIKV es causada por la picadura de un mosquito que, después de alimentarse de alguien contaminado, puede transportar el virus durante toda su vida y transmitir la enfermedad a una población que no tiene inmunidad. También se puede transmitir a través de la relación sexual de una persona con ZIKV a sus parejas, incluso si la persona infectada no tiene los sintomas de la enfermedad. En este trabajo, presentamos dos modelos matemáticos para la epidemia del Zika mediante el uso de (1) ecuaciones diferenciales ordinarias y, (2) ecuaciones diferenciales ordinarias con retardo temporal (discreto), que es el tiempo que tardan los mosquitos en desarrollar el virus. Hacemos una comparación entre las dos variantes de modelado. Se realizan simulaciones computacionales para Surinam y El Salvador, que son países propensos a desarrollar la epidemia de manera endémica.

Palabras clave. Ecuaciones diferenciales ordinarias, modelos, retardo, transmisión, ZIKV.

1. Introduction. Zika fever (also known as Zika virus disease) is an illness caused by the Zika virus. The disease is spread through the bite of daytime-active Aedes mosquitoes such as the A. aegypti and A. albopictus (these are the same mosquitoes that spread dengue and chikungunya viruses). Its name comes from Zika forest in Uganda, where the virus was first isolated from a rhesus monkey in 1947. The first human cases were reported in Nigeria in 1954. The first documented outbreak among people occurred in 2007, in the Federated State of Micronesia [15].

*ID ORCID: https://orcid.org/0000-0001-5937-5374, IME, University of São Paulo, Rua do Matão, 1010- CEP 05508090- São Paulo - SP (erickmath@ime.usp.br).

†ID ORCID: https://orcid.org/0000-0003-4823-0078, MATCOM, University of Habana, San Lázaro y L. Edificio Felipe Poey. Plaza de la Revolución, La Habana (aymee@matcom.uh.cu 
The disease of Zika virus is transmitted from infected Aedes mosquitoes to humans through mosquito bites [8]. It can also be transmitted from human to human through the blood and semen of an infected human, and through an infected pregnant woman to the foetus. Zika is a cause of microcephaly and other severe brain defects [4]. The incubation period (the time from exposure to symptoms) of Zika virus disease is not clear, but is likely to be a few days to a week. The symptoms are similar to other arbovirus infection such as dengue, and include fever, skin rashes, conjunctivitis (red eyes), muscle and joint pain, malaise and headache. These symptoms are usually mild and usually last from 2- 7 days [8].

There is no specific treatment or vaccine currently available for Zika virus disease. Prevention and control relies on reducing mosquitoes through source reduction (removal and modification of breeding sites), and reducing contacts between mosquitoes and people.

The use of ODE (ordinary differential equation) and ODE with delay in the study of epidemics can be seen in $[2,12]$, in particular for Dengue in [11, 18], for HIV/AIDS in [1, 19], for Ebola in [6] and Zika in [4, 15, 17], these texts contributed as background in the work that we present.

The objective of this work is to present models for the Zika epidemic based on ODE and ODE with delay. A theoretical study of the model was made and the $\Re_{0}$ was calculated for the sub-model with only contagion by mosquitoes and with only sexual contagion. Computational simulations are performed in Suriname and El Salvador, where ZIKV can become endemic. We performed a comparison between the two variants of modeling with respect to the time of epidemic and the number of infected.

2. Model with Exposed Variables . The model variables are susceptible men $H_{s}$, susceptible women $M_{s}$, exposed men $H_{E}$, exposed women $M_{E}$, infected men $H_{I}$, infected women $M_{I}$, recovered men $H_{R}$, recovered women $M_{R}$, susceptible mosquitoes $V_{s}$, exposed mosquitoes $V_{E}$ and infected mosquitoes $V_{I}$.

The Zika have a latent phase during which the individual is infected but not yet infectious. This delay between the acquisition of infection and the infectious state was incorporated within the model by adding a latent/exposed population, and letting infected (but not yet infectious) individuals move from susceptible to exposed and from exposed to infected.

The model is SEIR type (susceptible-exposed-infected-recovered) for humans and SEI (susceptible-exposed-infected) for mosquitoes, because mosquitoes do not recover.

All model parameters are assumed to be positive. Description of model (2.1) parameters are given in Table 2.1.

Assumptions for the construction of model:

- There is immunity in the recovered state, the infected man can infect women and men susceptible (result of the study of other epidemics that are transmitted by sexual contact).

- The death by natural causes is equal in any state, the death of mosquitoes will be due to environmental factors because no control strategy is applied.

- The $H_{s}, M_{s}, H_{E}, M_{E}, H_{I}, M_{I}, H_{R}, M_{R}, V_{s}, V_{E}$ and $V_{I}$ are continuous functions and positive or null (because we work with human and mosquitoes populations).

Let:

- The model is defined in an interval $\left[0, t_{f}\right]$, where $t_{f}$ is finite.

$\sigma_{v}:$ Number of times a single mosquito bites a human per unit time.

$\beta_{h v}$ : Probability of pathogen transmission from an infectious mosquito to a susceptible human given that a contact between the two occurs.

$\beta_{v h}$ : Probability of pathogen transmission from an infectious human to a susceptible mosquito given that a contact between the two occurs.

$N_{h}$ : Represent the total population of human.

Then,

$$
\begin{gathered}
\beta_{y_{1}}=\frac{\sigma_{v} \beta_{h v}}{N_{h}}=\frac{\beta_{y_{1 *}}}{N_{h}}, \\
\beta_{x}=\frac{\sigma_{v} \beta_{v h}}{N_{h}}=\frac{\beta_{x *}}{N_{h}} .
\end{gathered}
$$

To define $\beta_{y_{2}}$ and $\beta_{y_{3}}$ we did an analogous study but taking into account the sexual contacts (between men and heterosexual respectively) and the probability of infecting these contacts.

Let $l_{1}, l_{2}, l_{3}$ the life expectancy of men, women and mosquitoes. We define $\mu_{1}=\frac{1}{l_{1}}, \mu_{2}=\frac{1}{l_{2}}$ and $\eta=\frac{1}{l_{3}}$ such as death rates for men, women and mosquitoes respectively. 


\begin{tabular}{|l|l|}
\hline Parameters & Description \\
\hline$\beta_{y_{1}}$ & The force of infection from infected mosquito to susceptible human \\
\hline$\beta_{y_{2}}$ & The force of infection from infected man to susceptible man \\
\hline$\beta_{y_{3}}$ & The force of infection from infected man to susceptible woman \\
\hline$\beta_{x}$ & The force of infection from infected human to susceptible mosquito \\
\hline$\mu_{1}, \mu_{2}, \eta$ & Man, woman and mosquito mortality rate \\
\hline$\omega_{1}, \omega_{2}, \omega_{3}$ & $\begin{array}{l}\text { The rate of progression of men, women and mosquitoes from the exposed state to the } \\
\text { infectious state }\end{array}$ \\
\hline$\epsilon_{1}, \epsilon_{2}$ & Disease-induced death rate for humans (men and women) \\
\hline$r_{1}, r_{2}$ & Per capital recovery rate for humans from the infectious (men and women) \\
\hline$N_{1}, N_{2}, N_{3}$ & Recruitment rate of men, women and mosquitoes \\
\hline
\end{tabular}

TABLE 2.1

Description of parameters used in the model (2.1).

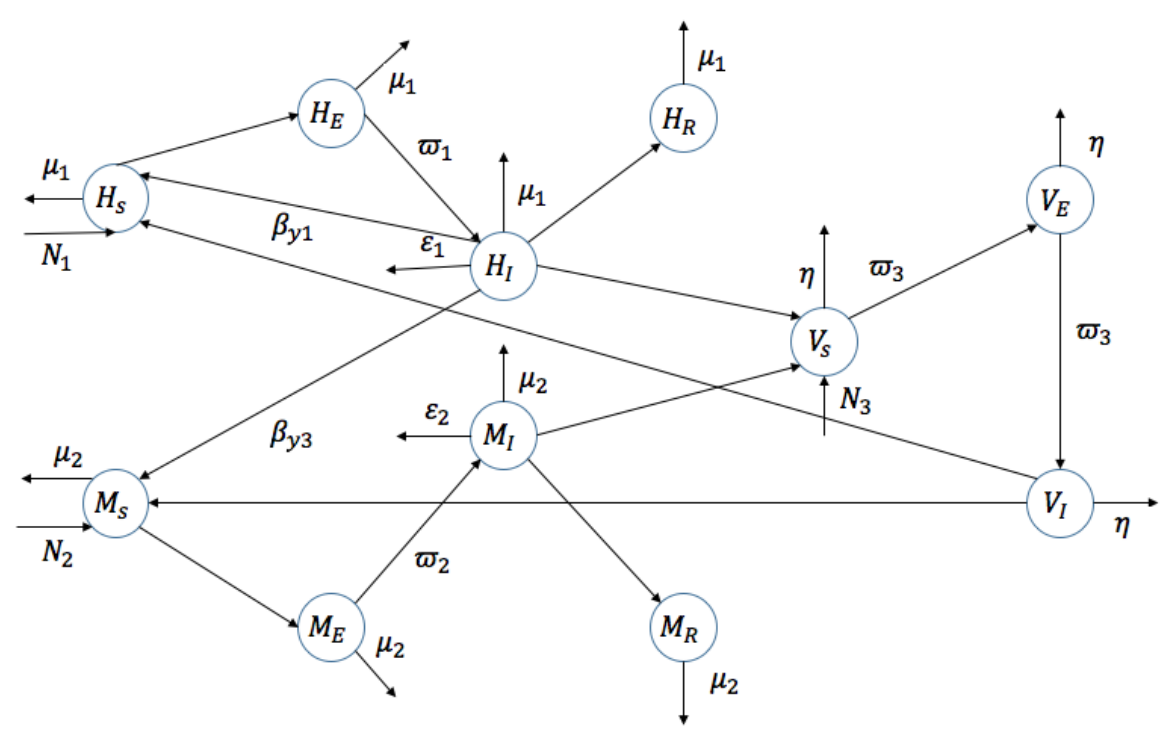

FIGURE 2.1. This schematic representation shows the progression of ZIKV in human and mosquito populations for our model. Susceptible humans start in $H_{s}, M_{s}$ (men and women) and move to $H_{E}$ and $M_{E}$, the exposed population, once infected by a mosquito carrying the virus and sexual contact. After an intrinsic incubation period, exposed individuals become infectious population and moves to the infectious population, $H_{I}$ and $M_{I}$. Infectious humans will then move to and remain in $H_{R}$ and $M_{R}$ after recovering from the infection. The susceptible mosquitoes population is denoted $V_{s}$. After transmission occurs from biting an infectious human, susceptible mosquitoes transition to the exposed population, $V_{E}$. The end of extrinsic incubation period marks the exposed mosquitoes shift to the infectious class $V_{I}$, where they remain infectious until death.

The transmission dynamics of the Zika is modeled by the system of ordinary differential equations (2.1). 


$$
\begin{aligned}
\frac{d H_{s}}{d t} & =N_{1}-\beta_{y_{1}} V_{I} H_{s}-\beta_{y_{2}} H_{I} H_{s}-\mu_{1} H_{s} \\
\frac{d M_{s}}{d t} & =N_{2}-\beta_{y_{1}} V_{I} M_{s}-\beta_{y_{3}} H_{I} M_{s}-\mu_{2} M_{s} \\
\frac{d H_{E}}{d t} & =\beta_{y_{1}} V_{I} H_{s}+\beta_{y_{2}} H_{I} H_{s}-\left(\omega_{1}+\mu_{1}\right) H_{E} \\
\frac{d M_{E}}{d t} & =\beta_{y_{1}} V_{I} M_{s}+\beta_{y_{3}} H_{I} M_{s}-\left(\omega_{2}+\mu_{2}\right) M_{E} \\
\frac{d H_{I}}{d t} & =\omega_{1} H_{E}-\left(\epsilon_{1}+\mu_{1}+r_{1}\right) H_{I} \\
\frac{d M_{I}}{d t} & =\omega_{2} M_{E}-\left(\epsilon_{2}+\mu_{2}+r_{2}\right) M_{I} \\
\frac{d H_{R}}{d t} & =r_{1} H_{I}-\mu_{1} H_{R} \\
\frac{d M_{R}}{d t} & =r_{2} M_{I}-\mu_{2} M_{R} \\
\frac{d V_{s}}{d t} & =N_{3}-\beta_{x} H_{I} V_{s}-\beta_{x} M_{I} V_{s}-\eta V_{s} \\
\frac{d V_{E}}{d t} & =\beta_{x} H_{I} V_{s}+\beta_{x} M_{I} V_{s}-\left(\omega_{3}+\eta\right) V_{E} \\
\frac{d V_{I}}{d t} & =\omega_{3} V_{E}-\eta V_{I}
\end{aligned}
$$

Initial Conditions

$$
\begin{array}{lrrr}
H_{s}(0)=h_{s}>0, & M_{s}(0)=m_{s}>0, & H_{I}(0)=h_{i}>0, \\
M_{I}(0)=m_{i}>0, & H_{R}(0)=h_{r} \geq 0, & M_{R}(0)=m_{r} \geq 0, \\
H_{E}(0)=h_{e} \geq 0, & M_{E}(0)=m_{e} \geq 0, & V_{s}(0)=v_{s}>0, \\
V_{I}(0)=v_{i}>0, & V_{E}(0)=v_{e} \geq 0 . &
\end{array}
$$

Model Analysis. Let:

$$
\begin{aligned}
H_{s}+H_{E}+H_{I}+H_{R} & =N, \\
M_{s}+M_{E}+M_{I}+M_{R} & =M, \\
V_{s}+V_{E}+V_{I} & =V .
\end{aligned}
$$

We begin by showing all feasible solutions are uniformly bounded in a proper subset of $\Omega$. The feasible region $\Omega$ with

$$
\Omega=\left\{\left(H_{s}, H_{E}, H_{I}, H_{R}, M_{s}, M_{E}, M_{I}, M_{R}, V_{s}, V_{I}, V_{R}\right) \in \Re_{+}^{11}: N \leq \frac{N_{1}}{\mu_{1}}, M \leq \frac{N_{2}}{\mu_{2}}, V \leq \frac{N_{3}}{\eta}\right\} .
$$

Differentiating both sides of (2.2), (2.3) and (2.4) with appropriate substitutions, we obtained the following differential equations:

$$
\begin{aligned}
N^{\prime} & =N_{1}-\mu_{1} N-\epsilon_{1} H_{I} \leq N_{1}-\mu_{1} N, \\
M^{\prime} & =N_{2}-\mu_{2} M-\epsilon_{2} M_{I} \leq N_{2}-\mu_{2} M, \\
V^{\prime} & =N_{3}-\eta V .
\end{aligned}
$$

Applying Grönwall Inequality in (2.5), (2.6) and (2.7), we obtained:

$$
\begin{aligned}
& N(t) \leq N(0) \exp \left(-\mu_{1} t\right)+\frac{N_{1}}{\mu_{1}}\left(1-\exp \left(-\mu_{1} t\right)\right), \\
& M(t) \leq M(0) \exp \left(-\mu_{2} t\right)+\frac{N_{2}}{\mu_{2}}\left(1-\exp \left(-\mu_{2} t\right)\right), \\
& V(t) \leq V(0) \exp (-\eta t)+\frac{N_{3}}{\eta}(1-\exp (-\eta t)) .
\end{aligned}
$$

where $N(0), M(0)$ and $V(0)$ represents the initial humans and mosquitoes population total.

Therefore, $0 \leq N \leq \frac{N_{1}}{\mu_{1}}, 0 \leq M \leq \frac{N_{2}}{\mu_{2}}$ and $0 \leq V \leq \frac{N_{3}}{\eta}$ as $t \rightarrow \infty$. This implies, $\frac{N_{1}}{\mu_{1}}$ is an upper bound for 
$N(t), \frac{N_{2}}{\mu_{2}}$ is an upper bound for, $M(t)$ and $\frac{N_{3}}{\eta}$ is an upper bound for $V(t)$ provided $N(0) \leq \frac{N_{1}}{\mu_{1}}, M(0) \leq \frac{N_{2}}{\mu_{2}}$ and $V(0) \leq \frac{N_{3}}{\eta}$.

Hence, all feasible solutions of model (2.1) enter the region $\Omega$ which is a positively invariant set. Thus, the system is biologically meaningful and mathematically well-posed in the domain of $\Omega$. In this domain, it is sufficient to consider the dynamics of the flow generated by the model system described by (2.1). Therefore, we summarized the results in the following lemma:

Lemma 1. The closed set $\Omega$ is positively invariant and attracting with respect to the model described by (2.1)

The existence, uniqueness and positivity was demonstrated using the theoretical results presented in [5, 14]

$\Re_{0}$ and Local Stability. The basic reproduction number, denoted $\Re_{0}$, is "the expected number of secondary cases produced, in a completely susceptible population, by a typical infective individual. If $\Re_{0}<1$, then on average an infected individual" produces less than one new infected individual over the course of its infectious period, and the infection cannot grow. Conversely, if $\Re_{0}>1$, then each infected individual produces, on average, more than one new infection, and the disease can invade the population [13].

The disease-free equilibrium point in the model is:

$$
v_{0}=\left(\frac{N_{1}}{\mu_{1}}, 0,0,0, \frac{N_{2}}{\mu_{2}}, 0,0,0, \frac{N_{3}}{\eta}, 0,0\right) .
$$

We use the theory presented in $[9,13]$ (next generation matrix method) to relate the local stability at the equilibrium point free of infection with the value of basic number of reproduction $\left(\Re_{0}\right)$.

To study the $\Re_{0}$, we will divide into two sub-models, (1) with sexual contagion $\left(\Re_{0}^{s}\right)$ and (2) with transmission by mosquito bites $\left(\Re_{0}^{m}\right)$, with the objective of interpreting the influence of these forms of transmission independently in the spread of ZIKV.

We eliminate all forms of sexual contagion and the sub-model with contagion by mosquito bites is:

$$
\begin{aligned}
\frac{d H_{s}}{d t} & =N_{1}-\beta_{y_{1}} V_{I} H_{s}-\mu_{1} H_{s}, \\
\frac{d M_{s}}{d t} & =N_{2}-\beta_{y_{1}} V_{I} M_{s}-\mu_{2} M_{s}, \\
\frac{d H_{E}}{d t} & =\beta_{y_{1}} V_{I} H_{s}-\left(\omega_{1}+\mu_{1}\right) H_{E}, \\
\frac{d M_{E}}{d t} & =\beta_{y_{1}} V_{I} M_{s}-\left(\omega_{2}+\mu_{2}\right) M_{E}, \\
\frac{d H_{I}}{d t} & =\omega_{1} H_{E}-\left(\epsilon_{1}+\mu_{1}+r_{1}\right) H_{I}, \\
\frac{d M_{I}}{d t} & =\omega_{2} M_{E}-\left(\epsilon_{2}+\mu_{2}+r_{2}\right) M_{I}, \\
\frac{d H_{R}}{d t} & =r_{1} H_{I}-\mu_{1} H_{R}, \\
\frac{d M_{R}}{d t} & =r_{2} M_{I}-\mu_{2} M_{R}, \\
\frac{d V_{s}}{d t} & =N_{3}-\beta_{x} H_{I} V_{s}-\beta_{x} M_{I} V_{s}-\eta V_{s}, \\
\frac{d V_{E}}{d t} & =\beta_{x} H_{I} V_{s}+\beta_{x} M_{I} V_{s}-\left(\omega_{3}+\eta\right) V_{E}, \\
\frac{d V_{I}}{d t} & =\omega_{3} V_{E}-\eta V_{I} .
\end{aligned}
$$

We calculate the Jacobian of the sub-model (2.8), and we build

$$
T=\left[\begin{array}{cccccc}
0 & 0 & 0 & 0 & 0 & \beta_{y_{1}} H_{s} \\
0 & 0 & 0 & 0 & 0 & 0 \\
0 & 0 & 0 & 0 & 0 & \beta_{y_{1}} M_{s} \\
0 & 0 & 0 & 0 & 0 & 0 \\
0 & \beta_{x} V_{s} & 0 & \beta_{x} V_{s} & 0 & 0 \\
0 & 0 & 0 & 0 & 0 & 0
\end{array}\right]
$$




$$
\Sigma=\left[\begin{array}{cccccc}
-\left(\omega_{1}+\mu_{1}\right) & 0 & 0 & 0 & 0 & 0 \\
0 & -\left(\epsilon_{1}+r_{1}+\mu_{1}\right) & 0 & 0 & 0 & 0 \\
0 & 0 & -\left(\omega_{2}+\mu_{2}\right) & 0 & 0 & 0 \\
0 & 0 & 0 & -\left(\epsilon_{2}+r_{2}+\mu_{2}\right) & 0 & 0 \\
0 & 0 & 0 & 0 & -\left(\omega_{3}+\eta\right) & 0 \\
0 & 0 & 0 & 0 & 0 & -\eta
\end{array}\right]
$$

which are the transmission and transition matrix respectively, using the methodology presented in [13].

The $-T \Sigma^{-1}$ is defined as the next generation matrix and the basic reproduction number with only mosquito contagion $\left(\Re_{0}^{m}\right)$ in $v_{0}$ is given by:

$$
\Re_{0}^{m}=\rho\left(-T \Sigma^{-1}\right)=\sqrt{k_{1}+k_{2}},
$$

where $\rho\left(-T \Sigma^{-1}\right)$ is the spectral radius of $-T \Sigma^{-1}$ matrix,

$$
k_{1}=\frac{\beta_{y_{1}} N_{1} \beta_{x} N_{3} \omega_{1} \omega_{3}}{\mu_{1} \eta^{2}\left(\omega_{1}+\mu_{1}\right)\left(\epsilon_{1}+\mu_{1}+r_{1}\right)\left(\omega_{3}+\eta\right)} \text { and } k_{2}=\frac{\beta_{y_{1}} N_{2} \beta_{x} N_{3} \omega_{2} \omega_{3}}{\mu_{2} \eta^{2}\left(\omega_{2}+\mu_{2}\right)\left(\epsilon_{2}+\mu_{2}+r_{2}\right)\left(\omega_{3}+\eta\right)} .
$$

We have the following lemma for (2.8):

Lemma 2. The disease-free equilibrium is locally asymptotically stable if $\Re_{0}^{m}<1$, and unstable if $\Re_{0}^{m}>1$ for the sub-model with only mosquito transmission.

The sub-model with sexual contagion is obtained assuming that the ZIKV is transmitted by sexual contact only (maintaining the considerations in the construction of model (2.1)). We eliminate the presence of mosquitoes in the model because they do not participate in the transmission dynamics.

$$
\begin{aligned}
\frac{d H_{s}}{d t} & =N_{1}-\beta_{y_{2}} H_{I} H_{s}-\mu_{1} H_{s}, \\
\frac{d M_{s}}{d t} & =N_{2}-\beta_{y_{3}} H_{I} M_{s}-\mu_{2} M_{s}, \\
\frac{d H_{E}}{d t} & =\beta_{y_{2}} H_{I} H_{s}-\left(\omega_{1}+\mu_{1}\right) H_{E}, \\
\frac{d M_{E}}{d t} & =\beta_{y_{3}} H_{I} M_{s}-\left(\omega_{2}+\mu_{2}\right) M_{E}, \\
\frac{d H_{I}}{d t} & =\omega_{1} H_{E}-\left(\epsilon_{1}+\mu_{1}+r_{1}\right) H_{I}, \\
\frac{d M_{I}}{d t} & =\omega_{2} M_{E}-\left(\epsilon_{2}+\mu_{2}+r_{2}\right) M_{I}, \\
\frac{d H_{R}}{d t} & =r_{1} H_{I}-\mu_{1} H_{R}, \\
\frac{d M_{R}}{d t} & =r_{2} M_{I}-\mu_{2} M_{R} .
\end{aligned}
$$

Using a methodology analogous to that applied to sub-model (2.8), we have to

$$
\begin{gathered}
T=\left[\begin{array}{cccc}
0 & \beta_{y_{2}} H_{s} & 0 & 0 \\
0 & 0 & 0 & 0 \\
0 & \beta_{y_{3}} M_{s} & 0 & 0 \\
0 & 0 & 0 & 0
\end{array}\right], \\
\Sigma=\left[\begin{array}{cccc}
-\left(\omega_{1}+\mu_{1}\right) & 0 & 0 & 0 \\
0 & -\left(\epsilon_{1}+r_{1}+\mu_{1}\right) & 0 & 0 \\
0 & 0 & -\left(\omega_{2}+\mu_{2}\right) & 0 \\
0 & 0 & 0 & -\left(\epsilon_{2}+r_{2}+\mu_{2}\right)
\end{array}\right] .
\end{gathered}
$$

are the transmission and transition matrices respectively and the basic reproduction number with only sexual contagion $\left(\Re_{0}^{s}\right)$ in $v_{0}$ is:

$$
\Re_{0}^{s}=\rho\left(-T \Sigma^{-1}\right)=\frac{\beta_{y_{2}} N_{1} \omega_{1}}{\mu_{1}\left(\omega_{1}+\mu_{1}\right)\left(\epsilon_{1}+\mu_{1}+r_{1}\right)} .
$$

We have the following result for (2.10):

Lemma 3. The disease-free equilibrium is locally asymptotically stable if $\Re_{0}^{s}<1$, and unstable if $\Re_{0}^{s}>1$ for the sub-model with only sexual transmission. 
3. ZIKV Model with Delay. The mosquito becomes infected when it consumes the blood of a sick person. Then, if the insect bites a healthy person, it transmits the virus, which enters the bloodstream and is incubated for 3 to 12 days, until the symptom begins appearance. The delay $\tau$ will refer to the time that the mosquito that delays in developing the pathogen, 4 to 7 days $[8,9]$. The delay is taken into account in the infected compartment and in the previous model this period was incorporated as exposed variables in humans and mosquitoes. The model is SIR type (susceptible-infected-recovered) for humans and SI (susceptible-infected) for mosquitoes.

The parameters, variables and initial conditions (taking into account the delay) maintain the definitions and restraints of the model (2.1).

The transmission dynamics of ZIKV taking into account the time delay is modeled by the system of differential equations with delay (discrete) following:

$$
\begin{aligned}
\frac{d H_{s}}{d t} & =N_{1}-\beta_{y_{1}} V_{I} H_{s}-\beta_{y_{2}} H_{I} H_{s}-\mu_{1} H_{s}, \\
\frac{d M_{s}}{d t} & =N_{2}-\beta_{y_{1}} V_{I} M_{s}-\beta_{y_{3}} H_{I} M_{s}-\mu_{2} M_{s}, \\
\frac{d H_{I}}{d t} & =\beta_{y_{1}} V_{I}(t-\tau) H_{s}+\beta_{y_{2}} H_{I} H_{s}-\left(\mu_{1}+r_{1}+\epsilon_{1}\right) H_{I}, \\
\frac{d M_{I}}{d t} & =\beta_{y_{1}} V_{I}(t-\tau) M_{s}+\beta_{y_{3}} H_{I} M_{s}-\left(\mu_{2}+r_{2}+\epsilon_{2}\right) M_{I}, \\
\frac{d H_{R}}{d t} & =r_{1} H_{I}-\mu_{1} H_{R}, \\
\frac{d M_{R}}{d t} & =r_{2} M_{I}-\mu_{2} M_{R}, \\
\frac{d V_{s}}{d t} & =N_{3}-\beta_{x} H_{I} V_{s}-\beta_{x} M_{I} V_{s}-\eta V_{s}, \\
\frac{d V_{I}}{d t} & =\beta_{x} H_{I} V_{s}+\beta_{x} M_{I} V_{s}-\eta V_{I} .
\end{aligned}
$$

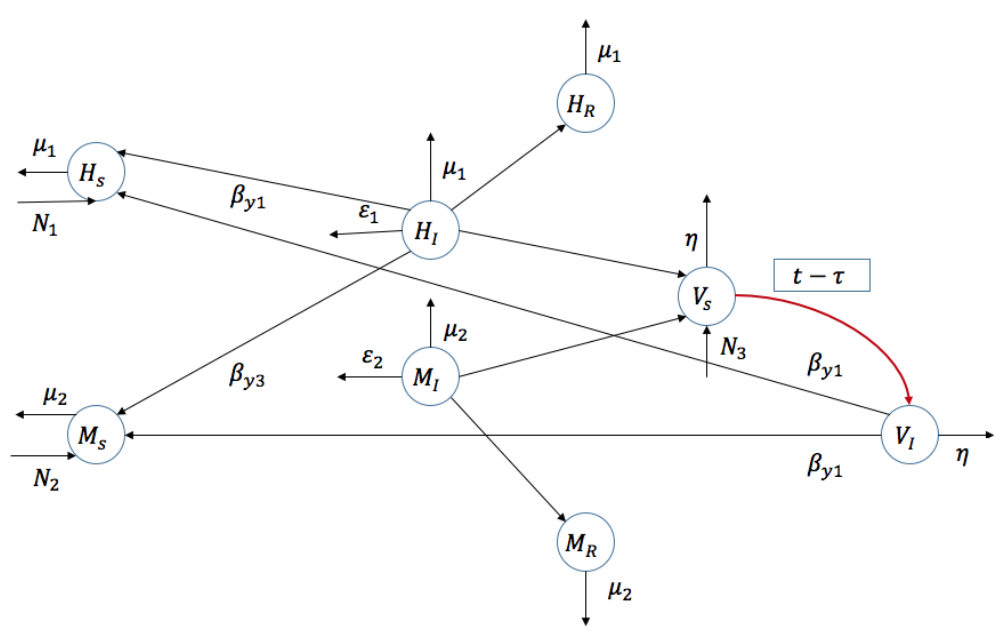

FIGURE 3.1. Schematic Representation of model with temporal delay.

Model Analysis. In this section we study the properties of the system solution (3.1) (existence, uniqueness and positivity).

Theorem 1. Let $f(t, x, y)$ and $f_{x}(t, x, y)$ be continuous on $\mathbb{R}^{n}, s \in \mathbb{R}$, and let $\phi:[s-r, s] \rightarrow \mathbb{R}$ be continuous. Then there exists $p>s$ and a unique solution of the initial-value problem (3.1) on $[s-r, p]$.

The enunciate and proof is in [10].

Let $f(t, x, y)=\left(F_{1}(t, x, y), F_{2}(t, x, y), \ldots, F_{8}(t, x, y)\right), x=\left(H_{s}, M_{s}, H_{I}, M_{I}, H_{R}, M_{R}, V_{s}, V_{I}\right)$ and $y=V_{I}(t-$ $\tau)$.

The $V_{I}(t-\tau)$ is continuous and positive function (by the form of construction of the model).

$F_{1}(t, x, y)=N_{1}-\beta_{y_{1}} V_{I} H_{s}-\beta_{y_{2}} H_{I} H_{s}-\mu_{1} H_{s}$,

$F_{2}(t, x, y)=N_{2}-\beta_{y_{1}} V_{I} M_{s}-\beta_{y_{3}} H_{I} M_{s}-\mu_{2} M_{s}$,

$F_{3}(t, x, y)=\beta_{y_{1}} V_{I}(t-\tau) H_{s}+\beta_{y_{2}} H_{I} H_{s}-\left(\mu_{1}+r_{1}+\epsilon_{1}\right) H_{I}$, 


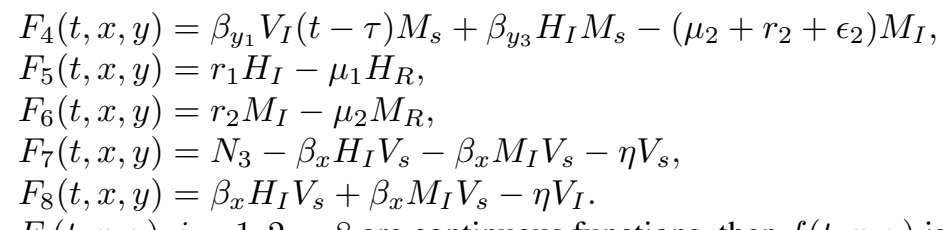

$F_{i}(t, x, y), i=1,2, . ., 8$ are continuous functions, then $f(t, x, y)$ is continuous.

$$
\begin{aligned}
& \frac{\partial F_{1}}{\partial H_{s}}=-\beta_{y_{1}} V_{I}-\beta_{y_{2}} H_{I}-\mu_{1}, \quad \frac{\partial F_{1}}{\partial H_{I}}=-\beta_{y_{2}} H_{s}, \quad \frac{\partial F_{1}}{\partial V_{I}}=-\beta_{y_{1}} H_{s}, \\
& \frac{\partial F_{1}}{\partial M_{s}}=\frac{\partial F_{1}}{\partial M_{I}}=\frac{\partial F_{1}}{\partial H_{R}}=\frac{\partial F_{1}}{\partial M_{R}}=\frac{\partial F_{1}}{\partial V_{s}}=0 . \\
& \frac{\partial F_{2}}{\partial M_{s}}=-\beta_{y_{1}} V_{I}-\beta_{y_{3}} H_{I}-\mu_{2}, \quad \frac{\partial F_{2}}{\partial H_{I}}=-\beta_{y_{3}} M_{s}, \quad \frac{\partial F_{2}}{\partial V_{I}}=-\beta_{y_{1}} M_{s}, \\
& \frac{\partial F_{2}}{\partial H_{s}}=\frac{\partial F_{2}}{\partial M_{I}}=\frac{\partial F_{2}}{\partial H_{R}}=\frac{\partial F_{2}}{\partial M_{R}}=\frac{\partial F_{2}}{\partial V_{s}}=0 . \\
& \frac{\partial F_{3}}{\partial H_{s}}=\beta_{y_{1}} V_{I}(t-\tau)+\beta_{y_{2}} H_{I}, \quad \frac{\partial F_{3}}{\partial H_{I}}=\beta_{y_{2}} H_{s}-\left(\mu_{1}+r_{1}+\epsilon_{1}\right), \\
& \frac{\partial F_{3}}{\partial M_{s}}=\frac{\partial F_{3}}{\partial M_{I}}=\frac{\partial F_{3}}{\partial H_{R}}=\frac{\partial F_{3}}{\partial M_{R}}=\frac{\partial F_{3}}{\partial V_{s}}=0, \frac{\partial F_{3}}{\partial V_{I}}=0 \text {. } \\
& \frac{\partial F_{4}}{\partial M_{s}}=\beta_{y_{1}} V_{I}(t-\tau)+\beta_{y_{3}} H_{I}, \quad \frac{\partial F_{4}}{\partial H_{I}}=\beta_{y_{3}} M_{s}, \quad \frac{\partial F_{4}}{\partial M_{I}}=-\left(\mu_{2}+r_{2}+\epsilon_{2}\right), \\
& \frac{\partial F_{4}}{\partial H_{s}}=\frac{\partial F_{4}}{\partial H_{R}}=\frac{\partial F_{4}}{\partial M_{R}}=\frac{\partial F_{4}}{\partial V_{s}}=\frac{\partial F_{4}}{\partial V_{I}}=0 . \\
& \frac{\partial F_{5}}{\partial H_{I}}=r_{1}, \quad \frac{\partial F_{5}}{\partial H_{R}}=-\mu_{1} \text {, } \\
& \frac{\partial F_{5}}{\partial H_{s}}=\frac{\partial F_{5}}{\partial M_{I}}=\frac{\partial F_{5}}{\partial M_{s}}=\frac{\partial F_{5}}{\partial M_{R}}=\frac{\partial F_{5}}{\partial V_{I}}=\frac{\partial F_{5}}{\partial V_{s}}=0 . \\
& \frac{\partial F_{6}}{\partial M_{I}}=r_{2}, \quad \frac{\partial F_{6}}{\partial M_{R}}=-\mu_{2}, \\
& \frac{\partial F_{6}}{\partial H_{s}}=\frac{\partial F_{6}}{\partial M_{s}}=\frac{\partial F_{6}}{\partial H_{I}}=\frac{\partial F_{6}}{\partial H_{R}}=\frac{\partial F_{6}}{\partial V_{s}}=\frac{\partial F_{6}}{\partial V_{I}}=0 . \\
& \frac{\partial F_{7}}{\partial H_{I}}=-\beta_{x} V_{s}, \quad \frac{\partial F_{7}}{\partial M_{I}}=-\beta_{x} V_{s}, \quad \frac{\partial F_{7}}{\partial V_{s}}=-\beta_{x} H_{I}-\beta_{x} M_{I}-\eta, \\
& \frac{\partial F_{7}}{\partial H_{s}}=\frac{\partial F_{7}}{\partial M_{s}}=\frac{\partial F_{7}}{\partial H_{R}}=\frac{\partial F_{7}}{\partial M_{R}}=\frac{\partial F_{7}}{\partial V_{I}}=0 . \\
& \frac{\partial F_{8}}{\partial H_{I}}=\beta_{x} V_{s}, \quad \frac{\partial F_{8}}{\partial M_{I}}=\beta_{x} V_{s}, \quad \frac{\partial F_{8}}{\partial V_{s}}=\beta_{x}\left(H_{I}+M_{I}\right), \quad \frac{\partial F_{8}}{\partial V_{I}}=-\eta, \\
& \frac{\partial F_{8}}{\partial H_{s}}=\frac{\partial F_{8}}{\partial M_{s}}=\frac{\partial F_{8}}{\partial H_{R}}=\frac{\partial F_{8}}{\partial M_{R}}=0 \text {. }
\end{aligned}
$$

$\left(F_{i}\right)_{x} i=1,2, \ldots, 8$ are continuous functions then $f_{x}$ is continuous.

The initial conditions are continuous and positive according to the model definition, so by Theorem 1 the solution of model is unique.

Theorem 2. Suppose that $f: \mathbb{R} \times \mathbb{R}_{+}^{n} \times \mathbb{R}_{+}^{n} \rightarrow \mathbb{R}^{n}$ satisfies the hypotheses of Theorem 1 and

$$
\forall i, t, \forall x, y \in R_{+}^{n}: x_{i}=0 \Rightarrow f_{i}(t, x, y) \geq 0 .
$$

If the initial data satisfy $\phi \geq 0$, then the corresponding solution $x(t)$ of (3.1) satisfies $x(t) \geq 0$ for all $t \geq s$ where it is defined, see [10].

$F_{1}\left(0, M_{s}, H_{I}, M_{I}, H_{R}, M_{R}, V_{s}, V_{I}\right)=N_{1}>0$,

$F_{2}\left(H_{s}, 0, H_{I}, M_{I}, H_{R}, M_{R}, V_{s}, V_{I}\right)=N_{2}>0$,

$F_{3}\left(H_{s}, M_{s}, 0, M_{I}, H_{R}, M_{R}, V_{s}, V_{I}\right)=\beta_{y_{1}} V_{I}(t-\tau) H_{s} \geq 0$,

$F_{4}\left(H_{s}, M_{s}, H_{I}, 0, H_{R}, M_{R}, V_{s}, V_{I}\right)=\beta_{y_{1}} V_{I}(t-\tau) M_{s}+\beta_{y_{3}} H_{I} M_{s} \geq 0$,

$F_{5}\left(H_{s}, M_{s}, H_{I}, M_{I}, 0, M_{R}, V_{s}, V_{I}\right)=r_{1} H_{I} \geq 0$,

$F_{6}\left(H_{s}, M_{s}, H_{I}, M_{I}, H_{R}, 0, V_{s}, V_{I}\right)=r_{2} M_{I} \geq 0$,

$F_{7}\left(H_{s}, M_{s}, H_{I}, M_{I}, H_{R}, M_{R}, 0, V_{I}\right)=N_{3}>0$,

$F_{8}\left(H_{s}, M_{s}, H_{I}, M_{I}, H_{R}, M_{R}, V_{s}, 0\right)=\beta_{x} V_{s}\left(H_{I}+M_{I}\right) \geq 0$.

The initial conditions are continuous and positive or null then by Theorem 2 the solution of model is positive.

4. Numerical Simulations and discussions. The objective of this subsection is to compare the two modeling techniques, with respect to the behavior of infected humans the time of epidemic and the outbreak period. Computational experimentation was carried out for Suriname and El Salvador because they are countries 
with characteristics that the ZIKV can become an endemic problem. The values used for the simulations (parameters and initial conditions) for each country are presented in the Tables 4.1, 4.2 and 4.3 and the delay $\tau$ is equal to 7 days. It was used for simulations Matlab2017a and to solve the system of ordinary differential equations, an adaptation of the ode45, which is based on an explicit Runge-Kutta formula, the DormandPrince pair. It is a one-step solver -for the solution in moment $n$, it needs only the solution at the immediately preceding time point, $n-1$. In general, ode45 is the best function to apply as a first try for most problems (https://www. mathworks.com/help/matlab/ref/ode45.html) and for the model with temporary delay, an adaptation of the routine DDE23, it tracks the discontinuities and integrates with the explicit pair of Runge-Kutta (2,3) and the interpolator of ode23. It uses iteration to take steps longer than the delays (https://www.mathworks.com/help/matlab/ref/dde23.html?s_tid=doc_ta).

\begin{tabular}{|c|c|c|}
\hline Parameters & Value & Reference \\
\hline$\beta_{y_{1}}$ & 0.1705 & {$[17]$} \\
\hline$\beta_{x}$ & 0.4352 & [17] \\
\hline$\beta_{y_{2}}$ & 0.003 & Assumed \\
\hline$\beta_{y_{3}}$ & 0.003 & Assumed \\
\hline$\omega_{3}$ & $\frac{1}{10.2}$ & [16] \\
\hline$\omega_{1}=\omega_{2}$ & $\frac{1}{6}$ & {$[11,4]$} \\
\hline$\mu_{1}=\mu_{2}$ & 0.0061 & https://www. indexmundi.com/g/g.aspx?c=ns\&v=26\&l=es \\
\hline$r_{1}=r_{2}$ & 0.55 & Assumed \\
\hline$\epsilon_{1}=\epsilon_{2}$ & 0.0004 & Assumed \\
\hline$\eta$ & $\frac{1}{18}$ & [15] \\
\hline$N_{1}$ & 0.65 & Assumed \\
\hline$N_{2}$ & 0.75 & Assumed \\
\hline$N_{3}$ & 0.60 & Assumed \\
\hline
\end{tabular}

Parameter values for Suriname

\begin{tabular}{|c|c|c|}
\hline Parameters & Value & Reference \\
\hline$\beta_{y_{1}}$ & 0.2808 & {$[17]$} \\
\hline$\beta_{x}$ & 0.3053 & [17] \\
\hline$\beta_{y_{2}}$ & 0.005 & Assumed \\
\hline$\beta_{y_{3}}$ & 0.007 & Assumed \\
\hline$\omega_{3}$ & $\frac{1}{10.2}$ & [16] \\
\hline$\omega_{1}=\omega_{2}$ & $\frac{1}{6}$ & {$[11,4]$} \\
\hline$\mu_{1}=\mu_{2}$ & 0.0057 & https://www. indexmundi. cboom/g/g.aspx?c=es\&v=26\&l=es \\
\hline$r_{1}=r_{2}$ & 0.75 & Assumed \\
\hline$\epsilon_{1}=\epsilon_{2}$ & 0.0004 & Assumed \\
\hline$\eta$ & $\frac{1}{18}$ & [15] \\
\hline$N_{1}$ & 0.65 & Assumed \\
\hline$N_{2}$ & 0.75 & Assumed \\
\hline$N_{3}$ & 0.60 & Assumed \\
\hline
\end{tabular}

Parameter values for El Salvador

\begin{tabular}{lcll}
\hline Variables & Suriname & El Salvador & Reference \\
\hline$H_{s}$ & 280.298 & 2.982 .221 & $\begin{array}{l}\text { https://www.datosmacro.com/demografia/poblacion/surinam } \\
\text { (surinam) }\end{array}$ \\
\hline$M_{s}$ & 278.070 & 3.362 .501 & $\begin{array}{l}\text { https://www.datosmacro.com/demografia/poblacion/el-salvador } \\
\text { (el-salvador) }\end{array}$ \\
\hline$H_{E}$ & 0 & 0 & Assumed \\
\hline$M_{E}$ & 0 & 0 & Assumed \\
\hline$H_{I}$ & 1000 & 1000 & Assumed \\
\hline$M_{I}$ & 1000 & 1000 & Assumed \\
\hline$H_{R}$ & 0 & 0 & Assumed \\
\hline$M_{R}$ & 0 & 0 & Assumed \\
\hline$V_{s}$ & 44222 & 152362 & {$[17]$} \\
\hline$V_{E}$ & 0 & 0 & Assumed
\end{tabular}


$\Re_{0}$ Study:

We calculate the $\Re_{0}^{m}$ for $\beta_{y_{1 *}} \in[0.04287,1.1241]$ (95 \% CI) in Suriname and $\beta_{y_{1 *}} \in[0.0119,0.9244]$ (95\% CI) in El Salvador (data extracted in [17]). The minimum value is 3.3932 and 6.3882 for El Salvador and Suriname respectively and shows that the infection will be able to spread in a population. The Figure 4.1 represents the growth of $\Re_{0}^{m}$ with respect to $\beta_{y_{1 *}}$ for Suriname and El Salvador.

The value of $\Re_{0}^{s}$ is 0.638643 and 0.793040 for Suriname and El Salvador respectively shows that the infection will die out in the long run. This form of contagion does not have a strong influence on the spread of the epidemic.
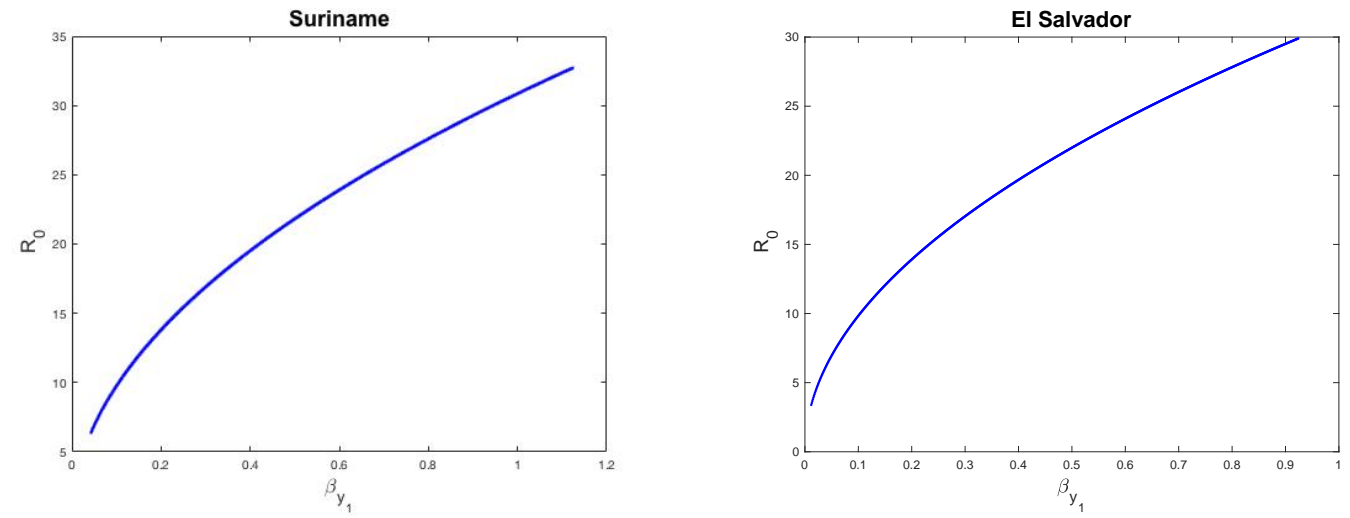

FIGURE 4.1. $\Re_{0}^{m}$ for Suriname and El Salvador. The basic reproduction number with only mosquito contagion (vertical axe) versus the product between the number of times a single mosquito bites a human per unit time and the probability of pathogen transmission from an infectious mosquito to a susceptible human given that a contact between the two occurs $\beta_{y_{1 *}}=\sigma_{v} \beta_{\text {hv }}$ (horizontal axe)

\section{Suriname:}

The model with temporal delay reports a greater number of infected people in the period in which the epidemic has greater force respect to model with exposed variables, see Figure 4.2.

For an approximate time of 45 days both models report the same number of men and women infected, see Figure 4.3, but at the end of a year, the model (2.1) reports a greater number of infected, see Figure 4.4. Throughout the year, the models report infected, demonstrating the endemic nature in these country if an adequate control strategy is not applied.
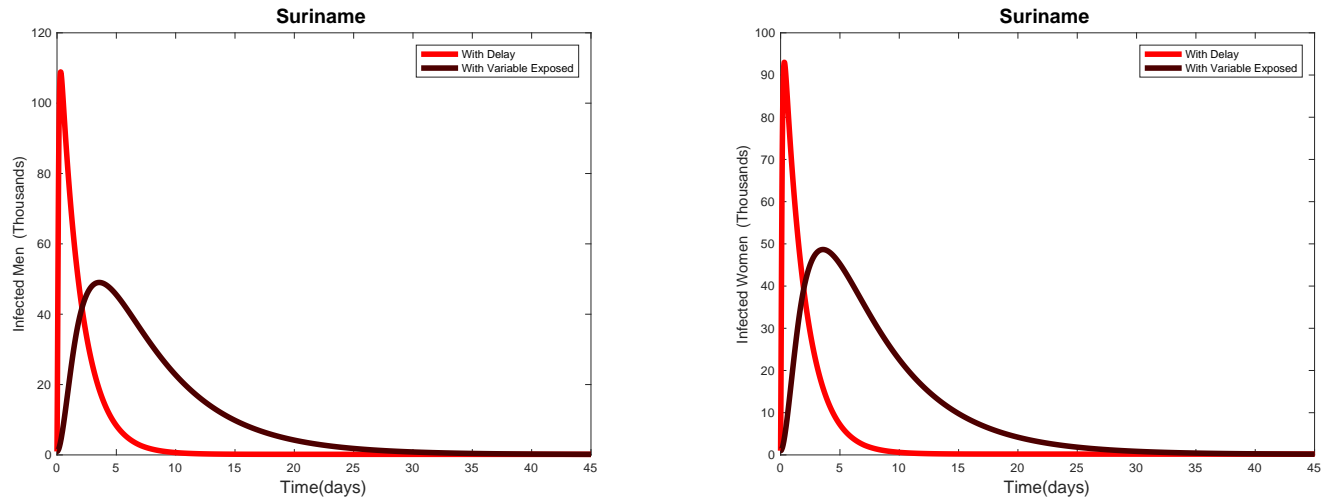

FIGURE 4.2. Comparison between models for Suriname. Number of infected by sex. Graphic of time versus infected number in thousands of people. 

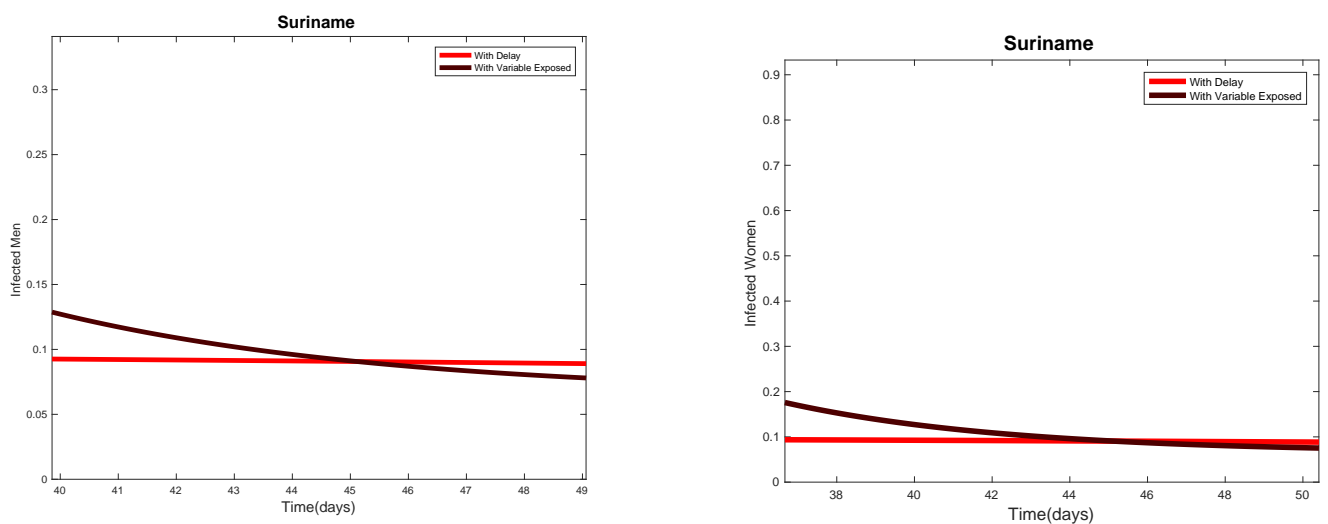

FIGURE 4.3. First moment of coincidence of the models for Suriname, men and women infected versus time.
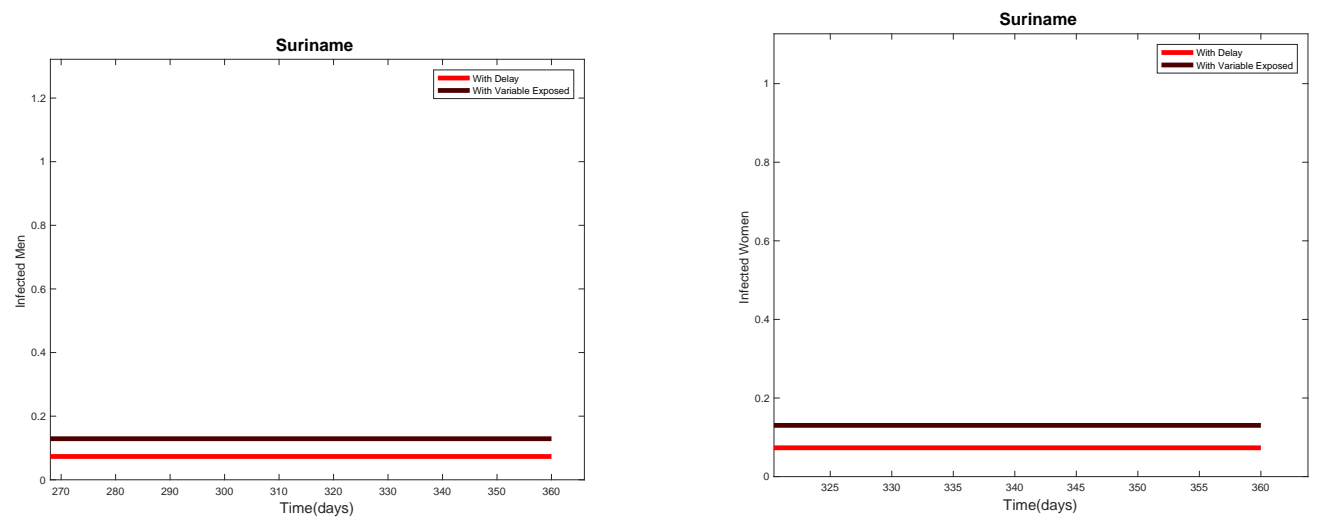

FIGURE 4.4. Number of infected reported by each model at the end of one year (approximately) for Suriname.

\section{El Salvador:}

The asymptotic behavior of infected humans is analogous to that of Suriname, see Figure 4.5. Both models report the same number of infected people after 50 days, a time longer than that reported for Suriname, but the population of El Salvador is greater than Suriname and the result obtained in the study of $\Re_{0}$ is verified because the ZIKV behaves as endemic, but contrary to Suriname, at the end of the period of time the model with delay reports a greater number of infected people compared to the model with exposed variables, see Figures 4.6 and 4.7.
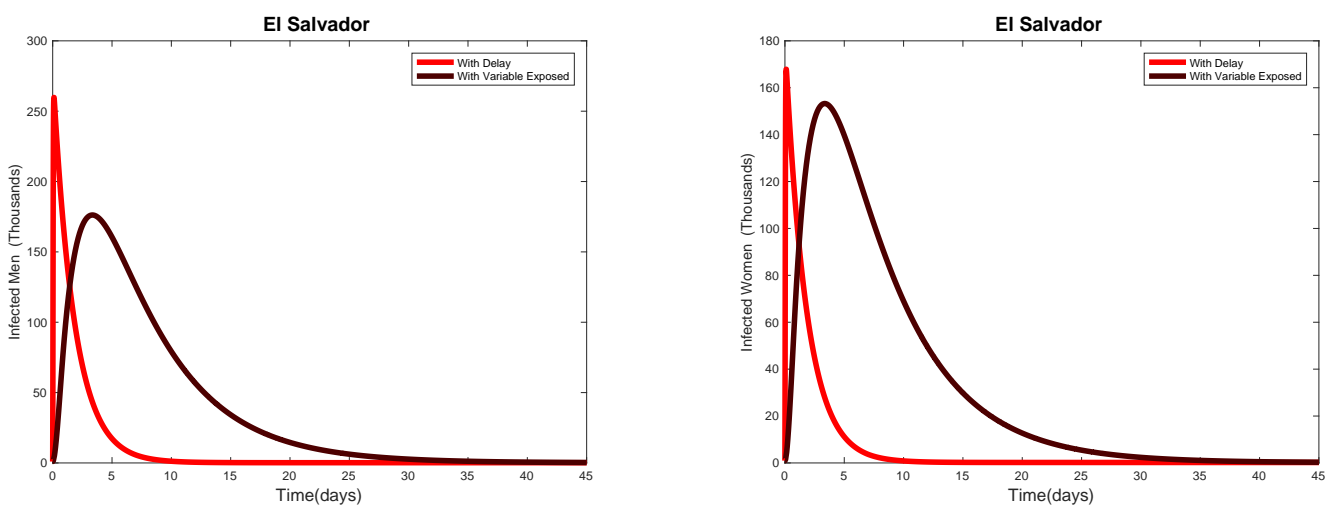

FIGURE 4.5. Comparison between models for El Salvador. Number of infected by sex. Graphic of time versus infected number in thousands of people. 

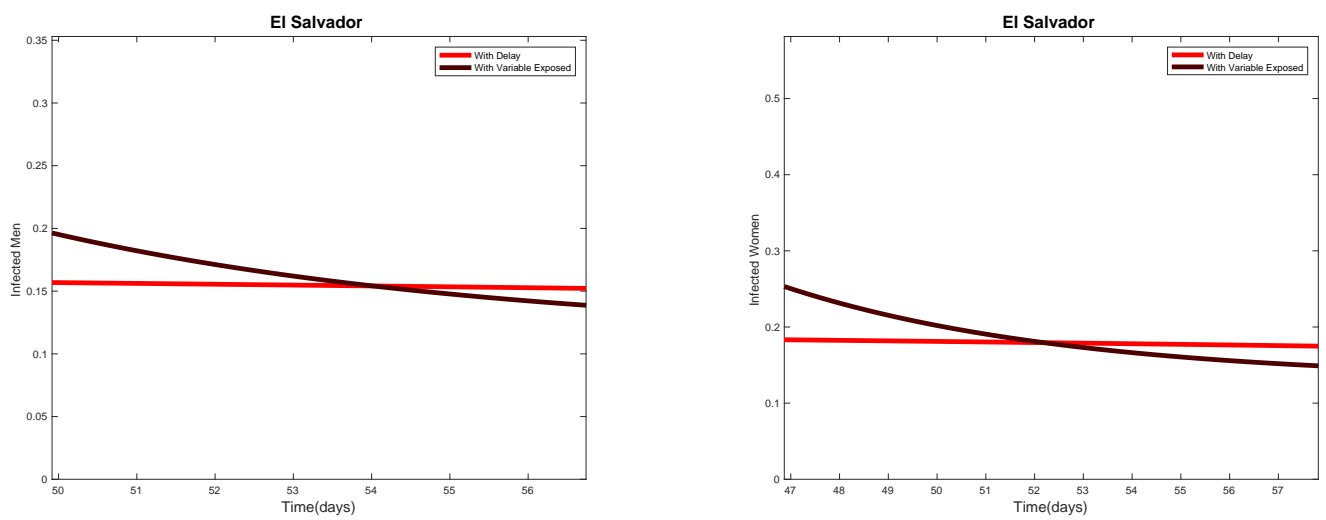

FIGURE 4.6. First moment of coincidence of the models for El Salvador. Men and women infected versus time.
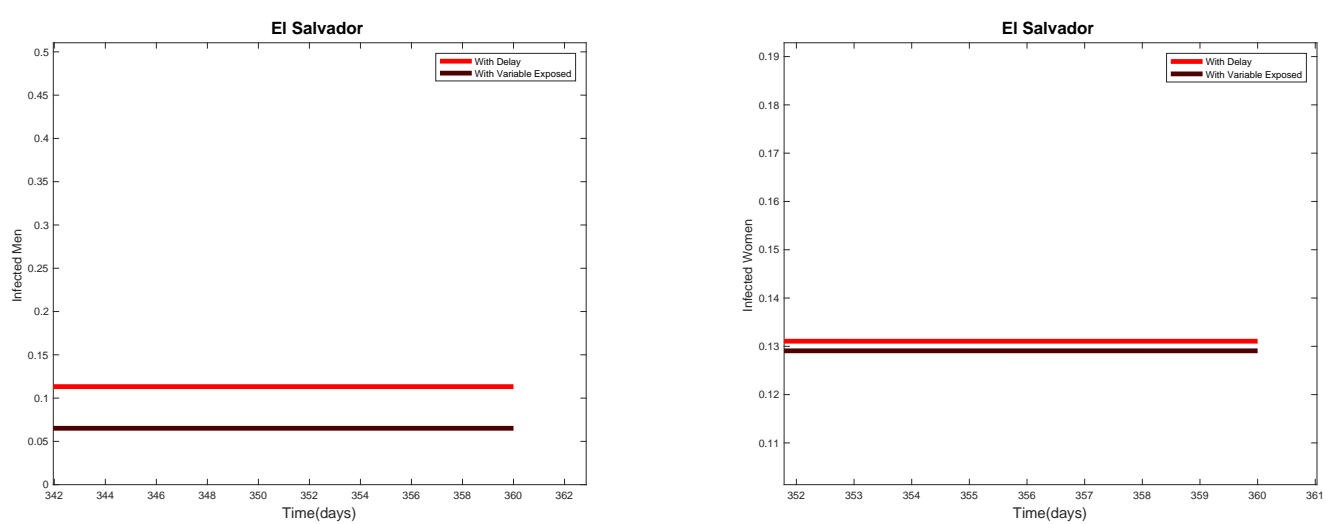

FIGURE 4.7. Number of infected reported by each model at the end of one year (approximately) for El Salvador.

5. Conclusions. We presented mathematical models for ZIKV with exposed variables and with temporal delay, and a comparison between them was made in relation to the period of the outbreak and the number of infected. The computational simulations were carried for El Salvador and Suriname because their demographic and geographic characteristics allow the study to be extended to other regions. The study for these countries showed that over time the Zika can become endemic and the need for a control strategy with priority in the contacts between mosquitoes and humans, because with the simulated data, sexual contagion does not exert a strong influence on the transmission of virus $\left(\Re_{0}^{s}<1\right)$. With the delay, the outbreak is reported for a shorter period of time but with a greater number of infected people compared to the model with the exposed variables for both countries.

\section{REFERENCES}

[1] Alizon, S and Magnus, C.; Modelling the course of an HIV infection: Insights from ecology and evolution, Viruses, 2: 1984-2013, 2012. ISSN: 1999-4915, DOI: 10.3390/v4101984.

[2] Badshah, V. H., Porwal, P. and Tiwari, V.; Mathematical modelling and role of dynamics in epidemiology, International Journal of Computational Science and Mathematics, 5(1): 4-10, 2013. ISSN: 0974-3189.

[3] Baker, C. T. H., Paul, C. A. H. and Willé, D. R.; Issues in the numerical solution of evolutionary delay differential equations, Adv. Comp. Math., 3: 171-196, 1995. DOI: 10.1016/S1474-6670(17)36928-8.ibitem, C.4 T. Berge, J. M. S. Lubuma,; . M. Moremedi, N. Morris and R. Kondera- Shava. A simple mathematical model for Ebola in Africa, Journal of Biological Dynamics, 11(1): 42-74, 2017. DO.1080/1 I: 10, 7513758.2016.1229817.

[4] Bonyah, E. and Okosun, k. C.; Mathematical modeling of Zika Virus, Asian Pacific Journal of Tropical Disease, 6 (9): 673-679, 2016. DOI: $0.1016 / \mathrm{S} 2222-1808(16) 61108$

[5] Chicone, C.; Ordinary differential equation with application, Springer Science and Business Media, 1999. ISBN: 0-387-30769-9.

[6] Deepa, O. S., Nallamalli, S., Naikand, L. N. S., Teja, G. V. S.; Mathematical model for transmission of Ebola, Procedia Computer Science, 48: 741-745, 2015. DOI: 10.1016/j.procs.2015.04.210.

[7] Dick, G. W. A., Kitchen, S. F. and Haddow, A. J.. Zika Virus. I. Isolations and serogical specifity, Trans R Soc Trop Med Hyg, 46(5): 509-520, 1952. DOI: 10.1016/0035-9203(52)90042-4.

[8] Dick, G. W. A., Kitchen, S. F. and Haddow, A. J.;, irus , G. V. S.;.Pathogenicity and physical properties, Trans R Soc Trop Med Hyg, 46: 521-534, 1952. DOI: 10.1016/0035-9203(52)90043-6.

[9] Diekmam, O.; Heesterbeek, J. A. P. and Roberts, M. G.. The construction of next- generation matrices for compartimental epidemical models, J. Royal Society Interface, 7: 873-885, 2010. DOI: 10.1098/rsif.2009.0386.

[10] Driver, R. D.; Ordinary and Delay Differential Equations. New York: Springer Verlag, 1977. ISBN: 0-387-90231-7. 
[11] Esteva, L. and Vargas, C.. Analysis of a Dengue disease transmission model, Mathematical Biosciences, 150: 131-151, 1998. DOI: 10.1016/S0025-5564(98)10003-2.

[12] Keeling, M. J. and Danon, L.. Mathematical Modelling of infectious diseases, Brithsh Medical Bulletin, 92(1): 33-42, 2009. DOI: 10.1093/bmb/ldp038.

[13] Van den Driessche, P. and Watmough, J.; Reproduction numbers and sub-threshold endemic equilibria for compartmental models of disease transmission, Mathematical Biosciences, 180: 29-48, 2002. DOI: 10.1016/S0025-5564(02)00108-6.

[14] Mattheij, R. and Molenar, J.. Ordinary differential equations in theory and practice, Published by Society for Industrial and Applied Mathematic, 2002. ISBN-13: 978-0898715316.

[15] Oluyo, T. O. and Adeyemi, M. O.. Mathematical analysis of Zika epidemic model, IOSR-JM, 12 (6): 21-33, 2017. ISSN: 2278-5728.

[16] Tiemi, T. T., Maidan, N. A., Ferreira JR, W. C., Paulino, P. and Yang, H.. Mathematical models for the aedes aegypti dispersal dynamics: Travelling waves by wing and wind, Bulletin of Mathematical Biology, 67: 509-528, 2005.

[17] Shutt, D. P., Manore, C. A., Pankavich, S. Porter, A. T. and Del Valle, S. Y.. Estimating the reproductive number, total outbreak size, and reporting rates for ZIKA epidemics in South and Central America, Epidemics, 21: 63-79, 2017. DOI: 10.1016/j.epidem.2017.06.005.

[18] Supriatna, A. K., Soewono, E. and Vangils, S.. A two- ages classes of Dengue transmission model, Math. Biosc., 216: 114-121, 2008. DOI: 10.1016/j.mbs.2008.08.011.

[19] Wordarz, D. and Nowak, M. A. Mathematical model of HIV pathogenesis and treatment, Bio ESSAYS, 24: 1178-1187, 2014. DOI: 10.1002/bies.10196 